\title{
Pedagogía de la formación doctoral: ¿Quiénes son "los otros" en la elaboración de una tesis?
}

\author{
Doctoral education pedagogy: Who are "the others" in \\ the making of a dissertation?
}

Viviana Mancovsky ${ }^{*}$ Laura Colombo ${ }^{* *}$

Recibido: 13 de diciembre de 2021 Aceptado: 15 de enero de 2021 Publicado: 31 de enero de 2022

To cite this article: Mancovsky, V. y Colombo, L. (2022). Pedagogía de la formación doctoral: ¿Quiénes son "los otros" en la elaboración de una tesis? Márgenes, Revista de Educación de la Universidad de Málaga. 3(1), 105-114

DOI: http://dx.doi.org/10.24310/mgnmar.v3i1.13962

\section{RESUMEN}

En este ensayo, reflexionamos sobre algunas ideas claves para una pedagogía de la formación doctoral prestando especial atención a "los otros" que acompañan los aprendizajes del sujeto que se forma. Luego de presentar algunas generalidades sobre la formación doctoral hacemos foco en el papel clave que pueden tener los espacios que habilitan el intercambio entre tesistas para acceder a una construcción colectiva del conocimiento y permitir conectarse con el lado productivo de la soledad. Para ello, damos cuenta de la implementación de un dispositivo pedagógico centrado en la tarea compartida de revisar borradores relacionados con la investigación doctoral: los grupos de escritura. Concluimos reconociendo la importancia que los tesistas pares, "los otros", pueden desempeñar al convertirse en interlocutores significativos en el largo y complejo proceso formativo tendiente al desarrollo de la autonomía intelectual.

Palabras clave: pedagogía de la formación doctoral; revisión entre tesistas pares; grupos de escritura; círculos de escritura

\section{ABSTRACT}

In this essay we reflect on some key ideas for a doctoral education pedagogy paying special attention to the social relations that contribute to students' learning process. After presenting some broad ideas about the doctoral experience, we focus on the importance of promoting initiatives that encourage interactions with others. This does not only allow to collectively construct knowledge but also to connect with a productive side of the doctoral loneliness. We illustrate the aforementioned by describing a pedagogical initiative centered on peer revision of doctoral dissertation drafts: writing groups. We conclude by recognizing the important role that peers, "others" can play as meaningful conversational partners in the long and complex learning process that leads to the development of intellectual autonomy.

Keywords: doctoral pedagogy; dissertation peer review; writing groups; writing circles

"Viviana Mancovsky 0000-0001-8184-291X

Profesora-investigadora de la Escuela de Humanidades de la Universidad Nacional de San Martin (UNSAM), (Argentina) vmancovsky@yahoo.com.ar
**Laura Colombo 0000-0001-6026-4436

Consejo Nacional de Investigaciones Científicas y Técnicas-Instituto de Lingüística, Universidad de Buenos Aires, (Argentina) laura.colombo@conicet.gov.ar 


\section{INTRODUCCIÓN}

Los aprendizajes que supone un doctorado académico tienen como protagonista a un sujeto ${ }^{1}$ que decide continuar sus estudios e iniciar una formación que le demandará la apropiación de un conjunto de saberes encuadrados en la "formación para la investigación” (Moreno Bayardo, 2015). A su vez, dichos aprendizajes múltiples y variados se despliegan en la dimensión del largo plazo y traducen una transformación subjetiva que encierra la figura de "volverse autor". Es decir, se trata de un sujeto que se asume como creador de saberes nuevos, rigurosos y sistemáticos. El sentido genuino de una experiencia formativa ${ }^{2}$ (Larrosa, 2009) puede entreverse en esa transformación hacia la autonomía intelectual. Los doctorandos comienzan a dilucidar quiénes pueden ser en el mundo académico. Este desarrollo de la identidad, tanto autoral como investigativa, está imbricada en un conjunto de relaciones entre individuos y culturas disciplinares que acarrean valores y formas de ser y de hacer en las diferentes áreas de conocimiento.

Ahora bien, nos interesa explicitar un doble posicionamiento a partir de esta afirmación que enunciamos a modo de postulado o punto de partida. Por un lado, enmarcamos estas reflexiones en torno al sujeto y sus aprendizajes desde una pedagogía de la formación doctoral; por otro, el logro de la autonomía intelectual se da en un contexto institucional determinado pautado por un programa doctoral específico. Este último define: criterios de acceso y admisión, una estructura curricular de estudio, los requisitos de presentación de la tesis y su posterior lectura, a cargo de un jurado de expertos.

Desde estas dos lógicas, la pedagógica y la institucional, que funcionan a la manera de un mirador para delimitar y a su vez, profundizar nuestra reflexión, avanzamos en formular un interrogante que motiva nuestra escritura: ¿quiénes son los posibles “otros” que acompañan a un sujeto en un programa doctoral?

Este ensayo tiene como propósito exponer las ideas centrales de una pedagogía de la formación doctoral focalizando en las interacciones entre tesistas pares que se encuentran en situación de avanzar con la escritura de sus tesis. Asimismo, da cuenta de la implementación de un dispositivo pedagógico llamado "grupos de escritura" basado en la tarea de revisión entre pares en torno a borradores relacionados con la investigación doctoral de los participantes.

En un primer apartado, nos interesa presentar las generalidades de una línea de investigación centrada en la pedagogía de la formación doctoral y desde ella, pondremos el acento en el proce-

1 Dado que aún no hay un acuerdo acerca del uso lingüístico del "o/a" o de la "@” para denotar los géneros tomando en cuenta las recientes recomendaciones al respecto de la Real Academia de la Lengua Española, en este texto se da por sentada la orientación hacia el logro de la equidad en materia de género y se usará solo el tradicional masculino como genérico a los fines de hacer más fluida la lectura.

2 Siguiendo los aportes valiosos de Larrosa (2009) la comprensión de la noción de experiencia, vinculada a la de formación, necesita tomar distancia de dos posiciones epistemológicas. Una de ellas, científica, asocia "experiencia" con "experimento" a partir del intento por objetivarla, medirla y controlarla. La ciencia moderna captura la experiencia haciéndola objetiva y universalizable. La otra posición, sostenida por la filosofía clásica, piensa a la experiencia como un primer modo de conocimiento sensible del cual hay que alejarse para alcanzar el verdadero saber. Por el contrario, la experiencia, explica el autor, es concebida desde la lógica de lo que acontece, siempre es única y singular y no se relaciona con el "tener" sino con el "hacer" experiencia. Por ello, una persona con experiencia es una persona abierta a su propia transformación. De allí que, el efecto subjetivo de la experiencia sea la formación. 
so de aprendizaje del sujeto que se forma. Luego, nos detendremos en exponer algunas reflexiones introductorias sobre la problemática específica de la tan nombrada y "reconocida” soledad del tesista, expresada de distintos modos y maneras por el sujeto protagonista que realiza una tesis. A partir de este análisis, nos interesa resaltar el rol fundamental que pueden tener los espacios formativos que favorecen el ensayo de diferentes formas de ser y hacer con la escritura, junto con "otros" en situación similar para fomentar la construcción colectiva del conocimiento.

En un tercer apartado, nos proponemos describir el acompañamiento centrado entre tesistas pares a partir de presentar la implementación de un dispositivo específico centrado en grupos de escritura. En ellos, cada participante comparte una versión preliminar de un texto que está escribiendo para que el resto lo lea y ofrezca comentarios e interpretaciones posibles de sentido. La retroalimentación obtenida entre pares sirve para mejorar los borradores. Este dispositivo ayuda a materializar el necesario acompañamiento para el aprendizaje situado (Lave \& Wenger, 1991) de la escritura en el posgrado ya que, por un lado, acompaña e intenta mejorar, mediante la retroalimentación, el proceso de redacción de la tesis y, por el otro, acompaña y forma lectores y revisores críticos. Dicha experiencia de trabajo en grupo pone en relieve el lugar de "otros" en el proceso formativo doctoral destacando tanto la importancia de la construcción de relaciones horizontales como el acompañamiento y la contención emocional que la propuesta brinda.

Por último, en las conclusiones, dejamos planteados algunos interrogantes vinculados con la puesta en marcha del dispositivo de acompañamiento entre tesistas pares en el marco de una pedagogía de la formación doctoral.

\section{PEDAGOGÍA DE LA FORMACIÓN DOCTORAL: BREVE RECORRIDO INTRODUCTORIO}

La pedagogía de la formación doctoral es una línea de investigación que se centra en el estudio de los procesos formativos y en las prácticas de acompañamiento institucional y académico desde el punto de vista de los sujetos implicados. Particularmente, suele centrarse en el estudio de la relación director de tesis y tesista (Åkerlind \& McAlpine, 2017; Bastalich, 2017; Mancovsky, 2013, 2015, en prensa).

En líneas generales y recuperando su origen etimológico, la pedagogía pone el acento en el encuentro con la alteridad, en los sujetos que llevan a cabo procesos de aprendizaje y en el acompañamiento necesario que esto supone (Mancovsky, 2015, 2021 ). Se define, de manera concisa pero categórica, como la reflexión sobre la educación y la formación de los sujetos, a partir de los aprendizajes que llevan a cabo (Meirieu, 1992). Por ello, sostenemos que hay necesidad de reflexión pedagógica cada vez que un sujeto aprende. Si esta afirmación clave la trasladamos al campo de la formación doctoral, podemos relevar la existencia de múltiples saberes por aprender: disciplinares, según el campo específico del objeto de investigación; relativos una escritura académica en la cual, el tesista debe apropiarse de las exigencias de un género discursivo altamente codificado y a partir del cual, construye un nuevo "posicionamiento enunciativo" y concernientes al oficio del investigador y todo lo que ello supone al llevar a cabo las etapas de un proyecto investigativo. Se trata, entonces, de un conjunto de saberes a adquirir, en el marco de un programa doctoral específico que impone condiciones y exigencias en tanto marco institucional preciso. 
Si bien la pedagogía suele ser acotada a los aprendizajes de la infancia y al ámbito escolar, ¿por qué pensarla en el contexto de una formación que ofrece el máximo grado académico? Dicho de otro modo: ¿por qué consideramos y ponemos en relieve al proceso de aprendizaje en la instancia de formación de adultos en el nivel especializado de un doctorado? Al recuperar nuevamente los planteos de Meirieu (2005) encontramos una respuesta significativa. El autor sostiene que

[...] los aprendizajes más especializados, los 'más sabios', aunque se presenten en un momento de nuestras vidas donde ya hemos aprendido a enfrentar lo desconocido y a pactar con la angustia, necesitan sostén y acompañamiento. [...] Los aprendizajes de los adultos no están liberados milagrosamente de la parte de nuestra infancia que todos guardamos en nuestro interior ya que, justamente, aprender significa: nacer a otra cosa, cada vez; descubrir mundos que ignorábamos hasta el momento [...] (p.17)

Esta reflexión contempla la dimensión subjetiva que muchas veces suele ser invisibilizada o ser analizada solo a los ojos de quien protagoniza la formación doctoral: el sujeto en formación. Sin embargo, los sujetos no aprenden solos. En efecto, desde un enfoque sociocultural del aprendizaje y la actividad humana (Vygotsky, 1978), se conciben los procesos de aprendizaje como procesos de participación social (Lave \& Wenger, 1991). Por lo tanto, esa transformación subjetiva que involucra un "volverse autor", como se mencionó en la introducción, no se da de manera vertiginosa ni repentina. Volverse autor está muy lejos de ser una mera “internalización” por parte del sujeto de un conocimiento objetivo sobre el sistema de la lengua o de la escritura académica como un ente homogéneo y asible. Por el contrario, implica un proceso de participación gradual en contextos sociales específicos mediante el uso de determinadas prácticas discursivas. Así, el desarrollo de la identidad escritora se produce mediante la gradual participación en comunidades disciplinares de práctica3 (Colombo, 2012; Lave \& Wenger, 1991) las cuales, a su vez, se ven transformadas por quienes se inician en ellas. A nivel de posgrado, las comunidades de práctica, en las cuales se integran progresivamente los estudiantes al producir textos académicos, están atravesadas por las particularidades de cada campo disciplinar, lo cual pone de relieve la conexión intrínseca entre la escritura y la disciplina científica en la que se desarrolla. Por ello, debemos reconocer la complejidad de las interacciones involucradas en la actividad letrada. Esta última, remite a una serie de “procesos laminados” mediante los cuales, los estudiantes se representan no sólo la tarea a realizar a través la escritura sino también, la forma en que los textos son recibidos y usados por profesores, pares y otros actores (Prior, 1998). Dicha actividad abre espacios para "la (re) socialización de las prácticas discursivas, la puesta en primer plano de representaciones de disciplinaridad, y para la negociación de las trayectorias de participación en comunidades de práctica” (Prior, 1998, p. 32). Por tanto, en este "volverse escritor" debemos reconocer la relevancia de las relaciones sociales en las prácticas escritoras ya que, al producir textos, los estudiantes pasan a integrar una red social relativamente institucionalizada y con una historia sociocultural específica y dinámica.

3 Se entiende por tal a "un conjunto de relaciones que se dan entre personas, actividades y mundo en un tiempo determinado; a veces, de manera tangencial y superpuesta con otras comunidades de práctica” (Lave y Wenger, 1991, p. 98). Wenger (1998) complementa esta definición proponiendo tres dimensiones que deben estar presentes para que exista una comunidad de práctica: un compromiso mutuo, un objetivo conjunto y un repertorio compartido de rutinas, formas de actuar y significados y recursos lingüísticos. 


\section{3. ¿SOLO O ACOMPAÑADO?}

Al detenernos en los relatos que los tesistas construyen acerca de su experiencia formativa, distintas imágenes cobran vida a través de sus palabras y sus frases significativas: decisión, cuestionamiento, desafío, esfuerzo, ámbitos alterados de la vida cotidiana, crecimiento personal, reordenamiento de la experiencia profesional vista a través del tiempo, lo ya sabido y lo por saber, dudas, temor, adrenalina, sentimiento de desorientación (“estar perdido”), sentir la tesis en el cuerpo ("ser una tesis caminado"). Nos interesa detenernos en una de esas imágenes que se vuelve recurrente en el diálogo con los doctorandos: la sensación de soledad que lleva implícito el proceso de escribir una tesis.

En un artículo titulado "La soledad del tesista de fondo", Lhérété (2011) ofrece una analogía creativa e interesante para pensar la formación del doctorando. La autora compara la experiencia formativa del investigador con la situación del maratonista que debe mantener el ritmo y sostener el esfuerzo en las carreras de fondo. Estas pruebas exigen cubrir una gran distancia y el desafío resulta ser la capacidad de resistencia del maratonista. En el caso del tesista, la autora señala, además, la necesidad de considerar al sentimiento de soledad como un aspecto que forma parte del aprendizaje del oficio del investigador (Lhérété, 2011). Avanzando en el despliegue de una temporalidad, el doctorando es llevado a desarrollar sus propios recursos que le permitirán llegar a "la línea final". En este proyecto, sostiene la autora, puede descubrir que el reconocimiento de su trabajo, a través de la mirada de "los otros", puede ser un apoyo crucial. Esos "otros" no supone solo el acompañamiento de su director sino, el compartir con pares sus avances, sus dudas, sus aciertos y sus pequeños pasos hacia la meta final: la defensa de su tesis.

En coincidencia con estas reflexiones, Bartolini (2020) propone conceptualizar la soledad como portadora de dos dimensiones luego de analizar las trayectorias de quienes se doctoraron y quienes no culminaron sus estudios en cuatro programas doctorales pertenecientes a diferentes campos disciplinares. Por un lado, la soledad elegida puede ser productiva ya que es necesaria para explorar el propio mundo interior y conectarse con la creatividad. Por el otro, la soledad no deseada puede volverse algo padecido, doloroso y que coarte la posibilidad de avanzar en la producción sistemática de conocimiento. El análisis de un cuerpo de datos basado en treinta y cuatro entrevistas, realizado por la autora, indicó que las experiencias de soledad dolorosa son las que experimentan con más frecuencia quienes se embarcan en el viaje doctoral. Propone, entonces, que los programas doctorales se organicen de forma tal de promover instancias de soledad productiva y de construcción social de conocimiento a fin de fomentar mejores experiencias educativas en este nivel de posgrado.

\section{UN DISPOSITIVO DE ACOMPAÑAMIENTO ENTRE PARES BASADO EN LA REVISIÓN: GRUPOS DE ESCRITURA}

En este apartado, nos interesa describir los grupos de escritura como un dispositivo de acompañamiento doctoral centrado en la tarea de revisión de borradores escritos por los propios tesistas. Concebimos el término "dispositivo" como una organización de prácticas, medios y recursos que apuntan a "crear las condiciones" para que los sujetos que participan en esta instancia, 
alcancen determinados propósitos en situaciones concretas. Generalmente, desde el campo de la pedagogía de la formación, los objetivos responden a fines formativos vinculados con la apropiación de saberes y prácticas (Mancovsky, 2016).

Ahora bien, los grupos de escritura que aquí detallamos están orientados al mejoramiento de los textos y las prácticas de escritura de sus participantes a través de actividades de revisión colectiva ${ }^{4}$. Por tanto, en ellos, cada miembro comparte una versión preliminar de un texto que está escribiendo para que el resto de los integrantes ofrezca comentarios para mejorarlos. Es necesario aclarar que, si bien se ejerce la tarea de revisión en forma conjunta, no se comparte la autoría ya que, quien concurre al grupo, lo hace con su propio proyecto de escritura. De hecho, una de las consignas dadas a los participantes es que son ellos quienes deciden qué presentar ante sus compañeros, con el requisito de que los textos en proceso estén relacionados con su investigación doctoral. Esto se orienta a que los participantes trabajen con proyectos de escritura asumidos como propios, lo cual permite que la actividad de revisar con "otros" adquiera un sentido en el aquí y ahora, con un objetivo no impuesto por otro (Colombo \& Carlino, 2015). Así, en los grupos muchas veces se revisan capítulos de tesis pero también, proyectos de investigación, artículos científicos, ponencias, resúmenes, y cualquier otro tipo de texto que ayude al avance de la investigación doctoral.

Para permitir una lectura atenta y no imponer una extensión máxima a los textos presentados en el grupo, la retroalimentación se brinda en dos etapas. Primero, se intercambia el borrador enviándolo por correo electrónico o poniéndolo en una carpeta compartida en Google Drive para que los tesistas pares lo comenten en forma escrita y lo envíen/compartan con el autor. Luego, se realiza una reunión sincrónica (cara a cara o en línea) en la cual se discuten ambos, el texto y los comentarios brindados. Esta suerte de retroalimentación en dos tiempos permite que "los comentadores" puedan abordar el texto en profundidad antes de la puesta en común colectiva. Los intercambios en tiempo real, que se dan durante las reuniones, abren un espacio de socialización basado en el trabajo sobre y con los textos (Rodas, Colombo, Calle \& Cordero, 2021).

Sin embargo, para que los doctorandos puedan sacar provecho de su participación en los grupos de escritura hace falta la intervención de un facilitador. Este es quien inaugura los grupos creando las condiciones para el intercambio entre pares: es decir, articula al grupo con la tarea. $\mathrm{Su}$ rol es fundamental al inicio ya que, por un lado, es necesario que instale pautas de funcionamiento y herramientas para que la tarea de intercambiar y comentar textos se lleve a cabo en forma ordenada. Por otro, el facilitador guía y andamia la tarea de dar y recibir comentarios en forma colaborativa para que los intercambios sirvan no sólo para enriquecer los textos sino también las prácticas escriturales de sus autores.

Es función del facilitador que los textos abordados en los grupos no se "corrijan" sino que se "comenten" desde un posicionamiento no normativo (Colombo, Bruno \& Silva, 2020). Es decir,

4 Este apartado se basa en los resultados de diferentes investigaciones con perspectiva cualitativa que utilizan el diseño de estudio de casos (Creswell, 2009; Stake, 1995) Ilevadas a cabo por la segunda autora. Las mismas se centran en diferentes grupos de escritura integrados por estudiantes de posgrado y profesores de instituciones argentinas y ecuatorianas respectivamente (por ej., Colombo \& Álvarez, 2021; Colombo, Bruno \& Silva, 2021; Colombo, Iglesias, Kiler \& Saez, 2022; Colombo \& Rodas, 2020; Rodas, Colombo, Calle \& Cordero, 2021). 
se espera que los participantes no emitan juicios de valor ${ }^{5}$ sobre los textos, sino que abran, ante los autores, su experiencia de lectura. Compartan sus preguntas, sus sensaciones y sus interpretaciones de lo leído. Si bien esto puede parecer sencillo de llevar a cabo, la experiencia de implementar grupos de escritura desde hace unos nueve años indica lo contrario. En primer lugar, es necesario que el facilitador brinde a los participantes algunas herramientas conceptuales sobre los distintos tipos de comentarios a realizar: basados en aspectos estructurales o gramaticales del texto (por ejemplo, coherencia entre párrafos, uso de conectores discursivos, vocabulario específico, etc.), centrados en el contenido del escrito, relacionados con el destinatario final, entre otros. En segundo lugar, hace falta detener la mirada de quien va a comentar los textos hacia la forma en la que se expresan los comentarios. Esto cobra especial importancia ya que, quienes llegan a un doctorado, han recibido durante su trayectoria por todo el sistema educativo, "correcciones" y no "comentarios" marcando aciertos y desaciertos en sus aprendizajes. De hecho, algunos participantes reportaron que este saber-hacer en cuanto a la forma de realizar devoluciones de los textos leídos, les ha sido útil en otros ámbitos, por ejemplo: al corregir parciales en su rol de docente universitario, al comentar borradores de tesis de licenciatura o maestría y al evaluar artículos para revistas científicas. De este modo, es menester del facilitador andamiar y acompañar el ejercicio de prácticas al interior del grupo de escritura que, en última instancia, ayudan a que los participantes se desarrollen como escritores de comunidades discursivas específicas y como revisores mediante el trabajo colaborativo entre pares. Así, las acciones del facilitador se despliegan en un territorio de intercambio que implica dar lugar a lo que le pasa al grupo, estar atento a sus tiempos, sus dificultades, teniendo en cuenta sus ritmos y actitudes para que se avance hacia una mirada y una producción compartidas. Las prácticas letradas ejercidas en los grupos, tanto la de producir como la de comentar escritos académicos, son constitutivas del proceso de publicación de artículos científicos. Es por esto por lo que los grupos de escritura, además de ayudar a avanzar con las tesis, constituyen un dispositivo de enseñanza de las prácticas letradas asociadas con la labor investigativa.

Además de articular al grupo con la tarea, el facilitador debe posicionarse de determinada manera durante el devenir grupal. Esto se relaciona, principalmente, con su modo de estar presente en las diferentes etapas del proceso de cada grupo. Si bien al momento de inaugurarlos y en los inicios, su presencia es fundamental y hasta constitutiva al momento de encuadrar la tarea, el objetivo último es que los grupos logren funcionar en forma autónoma. Por ende, el facilitador debiera ir gradualmente reduciendo sus intervenciones para, en última instancia, dejar de ejercer su rol, sin que esto afecte la dinámica grupal. Al respecto, la habilidad que ejerce un facilitador está en su disponibilidad y en su capacidad de espera para reconocer los tiempos del grupo, proponer recursos y abordajes que orienten el proceso de pensamiento colectivo. En última instancia, lo que se pretende poner en relieve en los grupos de escritura son las relaciones horizontales que dan lugar a diversos tipos de intercambios que enriquecen a los tesistas y a sus tesis.

5 Concebimos al juicio de valor como "una expresión verbal o no verbal formulada por un sujeto que estima la cualidad de un objeto o de otro sujeto" (Mancovsky, 2004, p. 11). Más precisamente, los juicios, en tanto enunciados apreciativos, positivos y/o negativos, se subordinan a la estructura normativa del lenguaje verbal y no-verbal. Asimismo, la noción de valor, considerada como cualidad y estimación, encierra la conformación de grados, en vistas a alcanzar un lugar determinado puesto como ideal (Mancovsky, 2004). 
Es, precisamente, en el intercambio con pares donde reside la riqueza de los grupos de escritura. En ellos, los participantes se brindan apoyo, ánimo y ayuda en forma sostenida, en pos de su progreso académico. En estos espacios, estén o no sus integrantes inscriptos institucionalmente en los mismos programas doctorales, son frecuentes los intercambios de materiales de diferente tipo que pueden allanar la experiencia doctoral. Por ejemplo, contar con documentos de circulación interna tales como planes de tesis, índices comentados o informes de avance es de utilidad ya que, estos modelos textuales ayudan a conceptualizar mejor la tarea de escritura. Así, desmitifican el desafío de producir determinado género discursivo, volviendo la tarea más plausible de ser llevada a cabo. En forma similar, al ir familiarizándose con los temas investigados por sus compañeros, los participantes suelen recomendarse bibliografía que podría ser insumo para elaborar los antecedentes o contribuir al armado del marco teórico de la investigación.

Otro aspecto que permite esta horizontalidad de las relaciones en los grupos de escritura, que, a su vez, justifica la retirada del facilitador, es la posibilidad de construcción de un ámbito de trabajo íntimo. Tener disponible un espacio cuidado donde expresar y compartir con "otros" las emociones experimentadas con respecto a la trayectoria doctoral y este "volverse autor" ligado a la tarea de investigar, ayuda a combatir los sentimientos de soledad antes mencionados. Encontrarse con "otros” que también están realizando sus estudios doctorales permite a los doctorandos poner en perspectiva su propia trayectoria. En este sentido, escuchar que a “otros” también les resulta trabajoso avanzar con la elaboración de la tesis, ayuda a que los doctorandos dejen de pensar que son ellos el obstáculo y se aminoren sus sentimientos de inseguridad. Esto les permitiría, entonces, alcanzar una soledad elegida y productiva, que los aleje del sufrimiento y los acerque a un posicionamiento introspectivo que motive su propia creatividad (Bartolini, 2020).

Por otro lado, la labor colaborativa alrededor de la producción intelectual y textual ayuda a promover e instalar nuevas formas de encarar la investigación que abren paso a la construcción social del conocimiento. En este sentido, esta formación contrarresta las lógicas individualistas, confrontativas y competitivas, muchas veces fomentadas por el mundo institucional académico-científico. Los intercambios genuinos entre los participantes de los grupos de escritura se dan gracias a la consolidación de lo vincular en pos de las actividades de revisión. Pese a que, el facilitador puede contar con algunas estrategias para favorecer una buena comunicación anticipando posibles inconvenientes en el trabajo con los vínculos, la modalidad en que esta tarea se lleva a cabo, no siempre es nítida ni directa. Por lo tanto, la facilitación implica transitar avances y retrocesos, aciertos y desaciertos; en fin, todo lo que puede pasar cuando aflora la subjetividad de aquellos que se encuentran implicados. Asimismo, la experiencia dicta que un aspecto fundamental para consolidar lo vincular consiste, sobre todo, en sostener la participación. Esto posibilita hacer frente a la incomodidad que suele generar el sentirse expuesto cuando se comparten borradores con “otros". El trabajo sostenido a lo largo del tiempo es fundamental porque da lugar a vivenciar una construcción social y dialogada del conocimiento.

En definitiva, los intercambios materiales y simbólicos que se dan al interior de los grupos de escritura en torno a la tarea de comentar textos, brindan un acompañamiento a la formación para la investigación y ofrecen recursos para avanzar con los proyectos de tesis doctorales de los participantes. 


\section{A MODO DE CONCLUSIÓN}

A partir de la presentación detallada de este dispositivo pedagógico, basado en el intercambio y en la revisión de textos entre doctorandos, creemos responder a nuestro título sugerido al inicio y, a su vez, poner en relevancia los aprendizajes entre pares. En el marco de una pedagogía de la formación doctoral, que reconoce los múltiples y singulares aprendizajes que se despliegan en la experiencia formativa del tesista, los pares pueden convertirse en interlocutores significativos en el largo y complejo desarrollo de la identidad tendiente hacia la autonomía intelectual.

Asimismo, los grupos de escritura se evidencian como un acompañamiento valioso que se centra en la construcción social de conocimiento y a su vez, en la elaboración individual de una tesis doctoral. Experiencia formativa siempre singular y única que se enriquece del intercambio con "otros" para fomentar la soledad productiva, en pos de la creación de saberes originales y rigurosos.

Además, más allá de la relación formativa enmarcada pedagógicamente entre director y tesista, este dispositivo da muestras de la valiosa complementariedad de los aprendizajes colaborativos propiciando el aprender a hacer comentarios cuidados entre pares. Esta reflexión pone de manifiesto la importancia de la construcción de relaciones de horizontalidad en el proceso de formación para la investigación, alejándose de posturas individualistas y competitivas que suelen aparecer en el ámbito académico-científico.

Por todas estas conclusiones, sostenemos el carácter eminentemente pedagógico de este dispositivo de acompañamiento centrado en la revisión de textos entre tesistas.

Por último, nos interesa dejar planteados algunos interrogantes que pueden servir como líneas de reflexión futuras para profundizar y afianzar la puesta en marcha de dicho dispositivo. En este sentido, nos preguntamos acerca del marco institucional más adecuado y pertinente para crear e instalar los grupos de escritura en los programas de formación doctoral. También, nos planteamos los ajustes necesarios para afinar el rol del facilitador en su modo de "inaugurar - estar - retirarse - seguir estando" según las etapas que atraviesa cada grupo específico de escritura. Finalmente, nos preguntamos acerca de la conformación más apropiada de los grupos, en función de las pertenencias institucionales y disciplinares diversas de los tesistas.

Decididamente, creemos que el espacio formativo que fomentan los grupos de escritura permite a los doctorandos no sólo compartir sus avances, dificultades, dudas, logros y aciertos en su trayectoria de la formación doctoral sino también, acompañarlos en el desafiante camino hacia la autonomía intelectual.

\section{REFERENCIAS}

Åkerlind, G., \& McAlpine, L. (2017). Supervising doctoral students: Variation in purpose and pedagogy. Studies in Higher Education, 42(9), 1686-1698. https://doi.org/1610.1080/03075079.03072015.01118031

Bartolini, A. M. (2020). Las soledades de los doctorandos. Una aproximación pedagógica Revista de Educación, 19, 165-185. https://bit.ly/3DRpCWY

Bastalich, W. (2017). Content and context in knowledge production: a critical review of doctoral supervision literature. Studies in Higher Education, 42(7), 1145-1157. https://doi.org/1110.1080/030 75079.03072015.01079702. 
Colombo, L. (2012). Escritura de posgrado y aprendizaje situado. En Facultad de Psicología de la Universidad de Buenos Aires (Ed.), Memorias del IV Congreso Internacional de Investigación y Práctica Profesional en Psicología - XIX Jornadas de Investigación - VIII Encuentro de Investigadores en Psicología del MERCOSUR [en CD] (Vol. 1, pp. 82-85). Buenos Aires, Argentina: Ediciones de la Facultad de Psicología - Universidad de Buenos Aires.https://bit.ly/31LWKlO

Colombo, L., \& Álvarez, G. (2021). Iniciativas didácticas basadas en la revisión entre pares y orientadas a la enseñanza-aprendizaje de la escritura de la tesis a nivel de posgrado. Educación y Humanismo, 23(40), 1-18. https://doi.org/10.17081/eduhum.17023.17040.13995.

Colombo, L., \& Carlino, P. (2015). Grupos para el desarrollo de la escritura científico-académica: Una revisión de trabajos anglosajones. Lenguaje, 43(1), 13-34. https://doi.org/10.25100/lenguaje. v25143i25101.24993

Colombo, L., Bruno, D., \& Silva, V. (2020). Grupos de escritura, vínculos y afectividad en el nivel de posgrado Praxis educativa, 24(3), 1-13. https://bit.ly/3rUySXP

Colombo, L., Iglesias, A., Kiler, M., \& Saez, V. (2022). Grupos de escritura en el postgrado: Experiencias de tesistas. Espacios en Blanco. Revista de Educación (Serie Indagaciones), 1(32), 163-172. https://ojs162. fch.unicen.edu.ar/ojs-163.161.160/index.php/espacios-en-blanco/article/view/870

Larrosa, J. (2009). Experiencia y alteridad en educacion. En: C. Skliar \& J. Larrosa. Experiencia y alteridad en educación (pp. 13-44). Homo Sapiens.

Lhérété, H. (2011). La solitude du thésard de fond. Revue Sciences Humaines. Octobre, 230, 48-50.

Mancovsky, V. (2004). La "des-naturalización” de la presencia permanente de los juicios de valor en el discurso del maestro. Revista del Instituto de Investigaciones en Ciencias de la Educación, 22,36-44. https://bit.ly/3pMcUna

Mancovsky, V. (2013). La dirección de tesis de doctorado tras las huellas de los saberes puestos en juego en la relación formativa. Revista Argentina de Educación Superior, 5(6), 50-71. https://bit.ly/3oJXK2s

Mancovsky, V. (2015) La formación para la investigación en programas doctorales. En V. Mancovsky \& M. G. Moreno Bayardo. La formación para la investigación en el posgrado (115-132). Novedades Educativas.

Mancovsky, V. (2016). Un dispositivo de intervención-investigación con directores de tesis de posgrado en las facultades de ciencias de la educación y de ciencias de la comunicación de la universidad de Málaga, desde una pedagogía doctoral. Revista del Instituto de Investigaciones en Educación, 7(9), 2942. https://bit.ly/3EMQRDf

Mancovsky, V. (Ed.)(2021). Pedagogía de la formación doctoral. Relatos vitales de directores de tesis. Biblos.

Meirieu, P.(1992). «Qui a peur des sciences del'éducation?» Revue Se former+.Pratiques et apprentissages de 1 'éducation. Lyon.

Meirieu, P. (2005). Lettre à un jeune professeur. ESF Éditeur.

Prior, P. (1998). Writing/disciplinarity: A sociohistoric account of literate activity in the academy. Lawrence Erlbaum Associates.

Rodas, E. L., Colombo, L., Calle, M. D., \& Cordero, G. (2021). Looking at faculty writing groups from within: some insights for their sustainability and future implementations. International Journal for Academic Development, https://doi.org/10.1080/1360144X.1362021.1976189.

Vygotsky, L. S. (1978). Mind in society: The development of higher psychological processes. Harvard University Press. 